\title{
Expansion of the hematopoietic stem cell compartment is necessary but not sufficient for gain-of-function mutant p53 R248Q to accelerate lymphomagenesis
}

\author{
Cell Death and Differentiation (2015) 22, 1397; doi:10.1038/cdd.2015.63; published online 5 June 2015
}

Dear Editor,

The importance of hematopoietic stem cells (HSCs) in the development of leukemias and lymphomas derived from mature lymphocytic cells is increasingly being recognized. ${ }^{1}$ Genetic and epigenetic changes occurring in the HSC compartment may either be maintained in differentiated cells and/or cooperate with other mutations that are acquired by differentiating cell populations. For example, HSCs isolated from CLL (chronic lymphocytic leukemia) patients show aberrancy in their subsequent differentiation characteristics, including skewed B-cell differentiation and generation of oligoclonal B-cell populations. ${ }^{2}$ Moreover, patients with T-cell lymphomas containing TET2 mutations were shown to carry this mutation already in early hematopoietic progenitors. ${ }^{3}$ These examples illustrate that some initiating events in lymphomagenesis may occur very early in hematopoietic differentiation.

Although wild-type p53 has an important role in HSC regulation, ${ }^{4}$ insights into how mutant p53 (mutp53) contributes to the initiation of lymphomagenesis remain elusive. Our recently generated mouse models probing in vivo effects of mutp53 show that the missense mutp53R248Q allele significantly accelerates T- and B-lymphomagenesis and shortens survival, compared with simple loss of p53 illustrating a potent gain-of-function (GOF) for this human hotspot mutation. ${ }^{5}$ Upon analyzing differentiation of $\mathrm{T}$ - and B-cells in mutp53R248Q mice, we found the stem cell compartment, comprised of HSCs and multipotential progenitors (MPPs) and isolated as LSK (Lin+Sca1+cKit+) FACS (fluorescence-activated cell sorting)-sorted population, was expanded by nearly $50 \%$ compared with p53nulls. ${ }^{5}$ This indicates that mutp53R248Q has a proproliferative GOF activity expanding the HSC/MPP population. ${ }^{5}$ (Parenthetically, the R248Q mesenchymal stem cell population was similarly expanded by $80 \%$ compared with p53nulls.) In contrast, mice expressing an alternative hotspot mutation, mutp53G245S, were identical to p53null mice in tumor latencies, survival and, notably, size of the HSC/MPP compartment. ${ }^{5}$

Here we ask whether the pro-proliferative GOF activity of the $\mathrm{R} 248 \mathrm{Q}$ allele within the uncommitted stem compartment (HSC/ MPP) is necessary and sufficient for accelerated T/B-lymphomagenesis, or alternatively, whether an additional tumorigenic GOF activity is at work. Hence, we generated floxed mutp53R248Q/mice (called floxQ/-), ${ }^{6}$ which exhibit identical GOF phenotypes as the constitutive mutp53R248Q. ${ }^{5}$ We crossed floxQ/- mice with p53 - / - ;CD2-iCre mice to generate floxQ/-;CD2-iCre and control p53-/ - ;CD2-iCre cohorts. CD2-iCre mice express Cre recombinase specifically at the common lymphocyte progenitor stage before splitting of T- and B-lineages. ${ }^{7}$ Thus, CD2-iCre restricts expression of R248Q to the HSC/MPP compartment, but abolishes its expression at the common lymphocyte progenitor stage and subsequent T/B-cell stages by converting the R248Q allele into p53null (Supplementary Figure $\mathrm{S} 1 \mathrm{~A})$. This allows us to isolate the effect that mutp53 exerts in the HSC/MPP compartment. Removing mutp53R248Q in pre-B cells and mature thymocytes while retaining it within the LSK compartment was confirmed by competitive PCR, and CD2-iCre-induced loss of mutp53 protein was confirmed by immunoblot of thymus and spleen lysates from floxQ/ - ;CD2-iCre mice (Supplementary Figure S1A). As expected, mutp53 was not expressed in T- and B-lymphomas, but remained expressed in solid tumors (Supplementary Figure S1B).

Surprisingly, we found no difference in tumor-free survival and overall survival between floxQ/-;CD2-iCre and p53-/ -;CD2-iCre mice. Moreover, there was no difference in latency of $T$ - and B-lymphomagenesis (Supplementary Figure S1C). Thus, upon conditional ablation of mutp53R248Q in committed lymphoid progenitors, the accelerated tumor onset GOF is lost.

Taken together with the lack of HSC/MPP expansion in mutp53G245S mice, ${ }^{5}$ our results identify a hitherto unknown twostage mechanism underlying acceleration of tumorigenesis in mutp53R248Q GOF mutants. Whereas the pro-proliferative GOF of mutp53R248Q in the HSC/MPP compartment is necessary for its expansion, an additional tumorigenic GOF occurring in lymphoid cells at the common lymphocyte progenitor or possibly later stages is required to cause acceleration. Whereas its specific molecular nature awaits elucidation, we recently showed that induced loss of mutp53R248Q from established tumors of floxQ/ - mice results in reduced aggressiveness and extended life span. ${ }^{6}$

\section{Conflict of Interest}

The authors declare no conflict of interest.

Acknowledgements. This work was supported by National Cancer Institute award 1R01CA176647 to UMM. We thank S Xu for technical assistance.

\section{AR Yallowitz ${ }^{1}$, W Hanel ${ }^{1}$ and UM Moll ${ }^{*, 1}$}

${ }^{1}$ Department of Pathology, Stony Brook University, Stony Brook, NY, USA

*Corresponding author: UM Moll, Department of Pathology, Stony Brook University, Stony Brook, NY 11794, USA. Tel: +1 631444 6816; Fax: +1 631444 3424; E-mail: Ute.Moll@stonybrookmedicine.edu

\footnotetext{
1. Jan M, Majeti R. Oncogene 2013; 32: 135-140.

2. Kikushige $Y$ et al. Cancer Cell 2011; 20: 246-259.

3. Quivoron C et al. Cancer Cell 2011; 20: 25-38.

4. Pant V, Quintas-Cardama A, Lozano G. Blood 2012; 120: 5118-5127.

5. Hanel W et al. Cell Death Differ 2013; 20: 898-909.

6. Alexandrova EM et al. Nature 2015; doi:10.1038/nature14430.

7. de Boer J et al. Eur J Immunol 2003; 33: 314-325.
} 